\title{
HILBERTIAN INTERPRETATIONS OF MANUALS
}

\author{
STANLEY P. GUDDER
}

\begin{abstract}
We characterize manuals which admit an interpretation in a manual on a Hilbert space. This characterization is given in terms of a certain set of states that the manual supports.
\end{abstract}

1. Introduction. In a series of papers $[1-4,8-10]$, D. Foulis and C. Randall developed a mathematical formalism for quantum mechanics and other empirical sciences. Their formalism is at a more primitive level than the quantum logic approach $[5,6,7,11]$ and, in fact, the latter can be derived from the former. In their approach, the physical operations form the basis of an axiomatic system in which the operations band together to form a mathematical structure called a manual.

A basic problem in axiomatic quantum mechanics is to characterize general quantum systems which are isomorphic to the traditional Hilbert space quantum mechanics. In this paper we give such a result for manuals. In particular, we show that a manual $A$ admits a Hilbert space morphism if and only if $A$ supports a collection of states of a certain type.

2. Definitions and notation. Most of the definitions in this section may be found in the work of Foulis and Randall cited in the introduction. Let $X$ be a nonempty set and let $A=A(X)$ be a collection of nonempty subsets of $X$ such that $X=\bigcup A$. The elements of $X$ are called outcomes, and the sets in $A$ are called operations. Any subset of an operation is an event. Denote the set of events by $\mathcal{E}(\mathcal{A})$. If $x, y \in E \in A$ and $x \neq y$ we write $x \perp y$. If $A \subseteq X$ we write

$$
A^{\perp}=\{x \in X: x \perp y \forall y \in A\}
$$

and for $A, B \subseteq X$ we write $A \perp B$ if $A \subseteq B \perp$. We call $A$ a manual if

(1) $E, F \in \bar{A}$ and $E \subseteq F$ implies $E=F$;

(2) $A, B \in \mathcal{E}(A)$ and $\bar{A} \perp B$ implies $A \cup B \in \mathcal{E}(A)$. that

A morphism $\phi$ from a manual $A$ to a manual $B$ is a map $\phi: \mathcal{E}(A) \rightarrow \mathcal{E}(B)$ such

(1) if $A_{i} \in \mathcal{E}(\mathcal{A})$ and $A=\bigcup A_{i} \in \mathcal{E}(\mathcal{A})$, then $\phi(A)=\bigcup \phi\left(A_{i}\right)$;

(2) if $A, B \in \mathcal{E}(A)$ and $A \perp \subseteq B \perp$, then $\phi(A) \perp \subseteq \phi(B) \perp$.

Let $\phi$ be a morphism from $A(X)$ to $B(Y)$. Then $\phi$ is said to be

(a) outcome preserving if $x \in X$ implies $\phi(x) \in Y$;

(b) operation preserving if $E \in \mathcal{A}$ implies $\phi(E) \in B$;

(c) faithful if $A, B \in \mathcal{E}(A)$ and $\phi(A) \perp \phi(B)$ implies $A \perp B$;

(d) conditioning if $A, B \in \mathcal{E}(A)$ and $A \perp B$ implies $\phi(A) \perp \phi(B)$;

Received by the editors May 11, 1981 and, in revised form, September 25, 1981.

1980 Mathematics Subject Classification. Primary 81B10; Secondary 06C15, 60A05, 46C10.

Key words and phrases. Manuals, interpretations, Hilbert space, quantum logic. 
(e) injective if $\phi$ is outcome preserving and $\phi(x)=\phi(y)$ implies $x=y$;

(f) a homomorphism if $\{\phi(E): E \in A\}$ is a manual;

(g) a homomorphism onto if $B=\{\phi(E): E \in A\}$;

(h) an isomorphism if $\phi$ is injective and a homomorphism onto $B$.

An operation preserving, conditioning morphism is an interpretation.

A state for a manual $\mathcal{A}$ is a map $\alpha: \mathcal{E}(\mathcal{A}) \rightarrow[0,1]$ such that

(1) if $E \in A$, then $\alpha(E)=1$;

(2) if $A, B \in \mathcal{E}(A)$ and $A \perp B$, then $\alpha(A \cup B)=\alpha(A)+\alpha(B)$.

A state $\alpha$ for $\mathcal{A}$ is regular if for any family $A_{i} \in \mathcal{E}(\mathcal{A})$ with $A_{i} \perp A_{j}, i \neq j$, such that $A=\bigcup A_{i} \in \mathcal{E}(A)$ we have $\alpha(A)=\sum \alpha\left(A_{i}\right)$. We denote the set of regular states on $A$ by $\sum(A)$. If $\alpha \in \sum(A), x \in X$, we write $\alpha(x)=\alpha(\{x\})$. It is clear that the function $x \rightarrow \alpha(x)$ determines $\alpha$. A function $f: X \rightarrow \mathbf{C}$ is an amplitude function [12] for $\alpha \in \sum(A)$ if $|f(x)|^{2}=\alpha(x)$ for all $x \in X$. Clearly, any $\alpha \in \sum(A)$ has many amplitude functions. Indeed, $f(x)=\lambda \alpha(x)^{1 / 2}$ is such a function for any $\lambda \in \mathbf{C}$ with $|\lambda|=1$. We call a function $f: X \rightarrow \mathbf{C}$ summable if $\sum_{x \in E} f(x)$ exists for all $E \in A$ and $\sum_{x \in E} f(x)=\sum_{x \in F} f(x)$ for all $E, F \in A$. An example of a summable function is $x \rightarrow \alpha(x)$ for any $\alpha \in \sum(A)$.

We now give an example of a manual which is of importance to our present work. Let $H$ be a complex Hilbert space and let $S(H)=\{v \in H:\|v\|=1\}$ be its unit sphere. Let $H_{1}$ be the set of all one-dimensional (orthogonal) projections on $H$ and let $A\left(H_{1}\right)$ be the collection of all maximal orthogonal sets in $H_{1}$. Then $A\left(H_{1}\right)$ is a manual. We call $A\left(H_{1}\right)$ the Hilbertian manual on $H$. Our Hilbertian manual is a submanual of the Foulis-Randall Hilbert space manual [4] consisting of the collection of all maximal orthogonal sets of projections on $H$. Both these manuals generate the same "operational logic" [4]. It is frequently convenient to consider the elements of $H_{1}$ as one-dimensional subspaces of $H$.

What distinguishes a Hilbertian manual $A\left(H_{1}\right)$ from among others in the general class of manuals? The Hilbertian manuals support a special set of states. Corresponding to a $v \in S(H)$ we define the vector state $\hat{v}$ by $\hat{v}(p)=\langle p v, v\rangle$ for all $p \in H_{1}$. Let $\mathcal{V}(H)=\{\hat{v}: v \in S(H)\}$. The set of vector states $\mathcal{V}(H)$ has two important properties. First, if $\emptyset \neq A \in \mathcal{E}(\mathcal{A})$, then there exists a $\hat{v} \in \mathcal{V}(H)$ such that $\hat{v}(A)=1$. For the second property, for each $p \in H_{1}$ choose a $\tilde{p} \in S(H)$ such that $\tilde{p} \in p$. If $E \in A\left(H_{1}\right)$, it is clear that $\{\tilde{p}: p \in E\}$ is an orthonormal basis for $H$. Now for each $\hat{v} \in \mathcal{V}(H)$ define the amplitude function $f_{v}: H_{1} \rightarrow \mathbf{C}$ by $f_{v}(p)=\langle v, \tilde{p}\rangle$. Then for any $\hat{u}, \hat{v} \in \mathcal{V}(H)$, the function $f_{u} \bar{f}_{v}$ is summable. Indeed, if $E, F \in A\left(H_{1}\right)$ we have

$$
\sum_{p \in E} f_{u}(p) \bar{f}_{v}(p)=\sum_{p \in E}\langle u, \tilde{p}\rangle\langle\tilde{p}, v\rangle=\langle u, v\rangle=\sum_{p \in F}\langle u, \tilde{p}\rangle\langle\tilde{p}, v\rangle=\sum_{p \in F} f_{u}(p) \bar{f}_{v}(p) .
$$

We close this section by defining families of states with certain special properties. Let $A(X)$ be a manual and let $\Delta \subseteq \sum(A)$. Then $\Delta$ is said to be

(1) unital if for any $\emptyset \neq A \in \mathcal{E}(\mathcal{A})$ there exists an $\alpha \in \Delta$ such that $\alpha(A)=1$;

(2) strongly separating if $x, y \in X$ and $x \neq y$ implies that there exists an $\alpha \in \Delta$ such that $\alpha(x) \neq \alpha(y)$;

(3) strongly $\perp$-determining if $\{\alpha \in \Delta: \alpha(A)=1\} \subseteq\{\alpha \in \Delta: \alpha(B)=0\}$ implies $A \perp B, A, B \in \bar{\varepsilon}(A)$;

(4) a set of amplitude states if for every $\alpha \in \Delta$ there is an amplitude function $f_{\alpha}$ for $\alpha$ such that for every $\alpha, \beta \in \Delta, f_{\alpha} \bar{f}_{\beta}$ is summable. 
We have shown above that $\mathcal{V}(H)$ is a unital set of amplitude states. It is also straightforward to show that $\mathcal{V}(H)$ is strongly separating and strongly $\perp-$ determining.

3. Hilbertian interpretations. We now characterize those manuals which have a Hilbertian interpretation.

THEOREM. A manual $A(X)$ has an outcome preserving interpretation $\phi$ in a Hilbertian manual $A\left(H_{1}\right)$ if and only if $A(X)$ admits a unital set of amplitude states $\Delta$. Moreover, if the above condition holds, then there exists an injection $\psi: \Delta \rightarrow$ $\mathcal{V}(H)$ such that $\alpha(A)=\psi(\alpha)[\phi(A)]$ for all $A \in \mathcal{E}(\mathcal{A})$.

Proof. Suppose $\phi: \mathcal{E}(A) \rightarrow \mathcal{E}\left[A\left(H_{1}\right)\right]$ is an outcome preserving interpretation. Then for every $A \in \mathcal{E}(\mathcal{A}), \phi(A)=\bigcup_{x \in A} \phi(x)$. For each $\hat{v} \in \mathcal{V}(H), A \in \mathcal{E}(\mathcal{A})$, define $\alpha_{v}(A)=\hat{v}[\phi(A)]=\sum_{x \in A} \hat{v}[\phi(x)]$. It is straightforward to show that each $\alpha_{v}$ is a regular state on $A(X)$. Let $\Delta=\left\{\alpha_{v}: \hat{v} \in \mathcal{V}(H)\right\}$. To show that $\Delta$ is unital, let $\emptyset \neq A \in \mathcal{E}(A)$ and let $p \in \phi(A)$. Then there exists a $v \in S(H)$ such that $v \in p$. Hence, $\alpha_{v}(A)=\hat{v}[\phi(A)]=1$. To show that $\Delta$ is a set of amplitude states, for each $p \in H_{1}$ choose a $\tilde{p} \in S(H)$ such that $\tilde{p} \in p$. For $\alpha_{v} \in \Delta$, define $f_{v}: X \rightarrow \mathbf{C}$ by $f_{v}(x)=\langle v, \phi(x) \sim\rangle$. Then $f_{v}$ is an amplitude function for $\alpha_{v}$ since

$$
\left|f_{v}(x)\right|^{2}=\mid\left\langle v,\left.\phi(x) \sim\right|^{2}=\hat{v}[\phi(x)]=\alpha_{v}(x) .\right.
$$

Finally, for any $E, F \in A(X), \alpha_{u}, \alpha_{v} \in \Delta$ we have

$$
\begin{aligned}
\sum_{x \in E} f_{u}(x) \bar{f}_{v}(x) & =\sum_{x \in E}\left\langle u, \phi(x)^{\sim}\right\rangle\left\langle\phi(x)^{\sim}, v\right\rangle=\langle u, v\rangle \\
& =\sum_{x \in F}\left\langle u, \phi(x)^{\sim}\right\rangle\left\langle\phi(x)^{\sim}, v\right\rangle=\sum_{x \in F} f_{u}(x) \bar{f}_{v}(x) .
\end{aligned}
$$

Conversely, let $\Delta$ be a unital set of amplitude states on $A(X)$ and let $H_{0}=$ $\left\{f_{\alpha}: \alpha \in \Delta\right\}$ be the corresponding set of amplitude functions. Let $\left[H_{0}\right]$ be the linear span of $H_{0}$. For $f, g \in\left[H_{0}\right]$ define $\langle f, g\rangle=\sum_{x \in E} f(x) \bar{g}(x)$ where $E \in A$. It is straightforward to show that $\langle f, g\rangle$ is well defined and independent of $E \in A$. It is clear that $\langle\cdot, \cdot\rangle$ is an inner product. Let $H$ be the Hilbert space completion of $\left[H_{0}\right]$ relative to the inner product $\langle\cdot, \cdot\rangle$. We now show that if $f \in H$, then $f: X \rightarrow \mathbf{C}$ and $\|f\|^{2}=\sum_{x \in E}|f(x)|^{2}=\sum_{x \in F}|f(x)|^{2}$ for every $E, F \in A$. Moreover, if $f, g \in H$, then $\langle f, g\rangle=\sum_{x \in E} f(x) \bar{g}(x)=\sum_{x \in F} f(x) \bar{g}(x)$ for every $E, F \in A$. Indeed, let $f_{i}$ be a Cauchy sequence in $\left[H_{0}\right]$. Then $f_{i}(x)$ converges in $\mathbf{C}$ for every $x \in X$ and hence there exists an $f: X \rightarrow \mathbf{C}$ such that $f_{i}(x) \rightarrow f(x)$ for all $x \in X$. It follows that $\left\|f-f_{i}\right\| \rightarrow 0$ as $i \rightarrow \infty$. Also,

$$
\begin{aligned}
\|f\|^{2} & =\lim \left\|f_{i}\right\|^{2}=\lim \sum_{x \in E}\left|f_{i}(x)\right|^{2}=\lim \sum_{x \in F}\left|f_{i}(x)\right|^{2} \\
& =\sum_{x \in E}|f(x)|^{2}=\sum_{x \in F}|f(x)|^{2} .
\end{aligned}
$$

The second statement now follows from the polarization identity.

For $x \in X$, let $\phi(x)$ be the linear span $\left[\left\{f_{\alpha}: \alpha(x)=1, \alpha \in \Delta\right\}\right] \subseteq H$. We now show that $\phi(x)$ is one-dimensional and hence in $H_{1}$. Suppose $\alpha(x)=\beta(x)=1$ for $\alpha, \beta \in \Delta$. If $y \perp x$, then $\alpha(y)=\beta(y)=0$ and hence, $f_{\alpha}(y)=f_{\beta}(y)=0$. Thus, if 
$x \in E \in A$ we have

$$
\left|\left\langle f_{\alpha}, f_{\beta}\right\rangle\right|=\left|\sum_{y \in E} f_{\alpha}(y) \bar{f}_{\beta}(y)\right|=\left|f_{\alpha}(x)\right|\left|f_{\beta}(x)\right|=1=\left\|f_{\alpha}\right\|\left\|f_{\beta}\right\| .
$$

Since we have an equality in Schwarz's inequality, there is a $\lambda \in \mathbf{C}$ such that $f_{\beta}=$ $\lambda f_{\alpha}$. Hence, $\phi: X \rightarrow H_{1}$ and $\phi$ preserves outcomes. Extend $\phi$ to $\mathcal{E}(A)$ by defining $\phi(A)=\bigcup_{x \in A} \phi(x)$ for $A \in \mathcal{E}(\mathcal{A})$. If $E \in A$, we now show that $\phi(E) \in \mathcal{A}\left(H_{1}\right)$. Let $x, y \in E$ with $x \neq y$ and suppose $\alpha(x)=\beta(y)=1, \alpha, \beta \in \Delta$. Then

$$
\left\langle f_{\alpha}, f_{\beta}\right\rangle=\sum_{z \in E} f_{\alpha}(z) \bar{f}_{\beta}(z)=\sum_{z \in E} \delta_{x z} \delta_{y z}=0 .
$$

Hence, $\phi(x) \perp \phi(y)$. Now let $p \in H_{1}$ satisfy $p \perp \phi(E)$. If $g \in p, x \in E$ and $\alpha(x)=1$, then $g \perp f_{\alpha}$. Hence,

$$
0=\left\langle g, f_{\alpha}\right\rangle=\sum_{y \in E} g(y) \bar{f}_{\alpha}(y)=g(x) \bar{f}_{\alpha}(x) .
$$

Hence, $g(x)=0$ for all $x \in E$ and $\|g\|^{2}=\sum_{x \in E}|g(x)|^{2}=0$. Thus, $g=0$. It follows that $\phi(E) \in A\left(H_{1}\right)$ so $\phi$ preserves operations and events. Hence, $\phi: \mathcal{E}(\mathcal{A}) \rightarrow$ $\mathcal{E}\left[\mathcal{A}\left(H_{1}\right)\right]$. Moreover, if $x \perp y$ then $\phi(x) \perp \phi(y)$ so $A, B \in \mathcal{E}(\mathcal{A})$ and $A \perp B$ implies $\phi(A) \perp \phi(B)$.

We now show that $\phi$ is a morphism and therefore an interpretation (actually this follows from [4, Lemma 2], but we shall give the proof to make this work selfcontained). First, condition (1) in the definition of a morphism clearly holds. For condition (2), suppose $A, B \in \mathcal{E}(A)$ and $A \perp \subseteq B \perp$. Let $p \in \phi(A) \perp$ and $q \in \phi(B)$ and let $f, g \in H$ satisfy $f \in p, g \in q$. Let $E \in A$ satisfy $A \subseteq E$. Then $E-A \subseteq$ $A \perp \subseteq B \perp$ and hence

$$
\phi(E-A) \subseteq \phi\left(B^{\perp}\right) \subseteq \phi(B) \perp \subseteq q^{\perp} .
$$

Hence $q \in \phi(E-A) \perp$. Since $\sum_{x \in E} \phi(x)=I$, we have $f=\left[\sum_{x \in E-A} \phi(x)\right] f$ and $g=\left[\sum_{x \in A} \phi(x)\right] g$. Hence, $f \perp g$ so $p \perp q$ and $\phi(A) \perp \subseteq \phi(B) \perp$.

For the last statement of the theorem, define $\psi: \Delta \rightarrow \mathcal{V}(H)$ by $\psi(\alpha)=\hat{f}_{\alpha}$. Let $x \in X$ and let $\beta \in \Delta$ satisfy $\beta(x)=1$. Then for any $\alpha \in \Delta$ we have

$$
\psi(\alpha)[\phi(x)]=\hat{f}_{\alpha}[\phi(x)]=\left\langle\phi(x) f_{\alpha}, f_{\alpha}\right\rangle=\left|\left\langle f_{\alpha}, f_{\beta}\right\rangle\right|^{2}=\left|f_{\alpha}(x)\right|^{2}=\alpha(x) .
$$

Hence, for any $A \in \mathcal{E}(A)$ we have

$$
\psi(\alpha)[\phi(A)]=\psi(\alpha)\left[\bigcup_{x \in A} \phi(x)\right]=\sum_{x \in A} \psi(\alpha)[\phi(x)]=\sum_{x \in A} \alpha(x)=\alpha(A)
$$

CoROllary. A manual $A(X)$ has an injective interpretation in a Hilbertian manual if and only if $A(X)$ admits a unital, strongly separating set of amplitude states.

ProOF. Suppose $\Delta$ is a unital, strongly separating set of amplitude states on $A(X)$. Let $\phi: X \rightarrow H_{1}$ and $\psi: \Delta \rightarrow \mathcal{V}(H)$ be the maps constructed in the theorem. To show that $\phi$ is injective assume that $\phi(x)=\phi(y)$. Then for any $\alpha \in \Delta$ we have

$$
\alpha(y)=\psi(\alpha)[\phi(y)]=\psi(\alpha)[\phi(x)]=\alpha(x) .
$$


Hence $x=y$. Conversely, suppose $\phi: \mathcal{E}(\mathcal{A}) \rightarrow \mathcal{E}\left[A\left(H_{1}\right)\right]$ is an injective interpretation in a Hilbertian manual $A\left(H_{1}\right)$. Define $\Delta=\left\{\alpha_{v}: \hat{v} \in \mathcal{V}(H)\right\}$ as in the theorem. The theorem shows that $\Delta$ is a unital set of amplitude states on $A$. To show that $\Delta$ is strongly separating assume that $\alpha(x)=\alpha(y)$ for all $\alpha \in \Delta$. Then $\hat{v}[\phi(x)]=\hat{v}[\phi(y)]$ for all $\hat{v} \in \mathcal{V}(H)$. Since $\mathcal{V}(H)$ is strongly separating on $A\left(H_{1}\right)$ we have $\phi(x)=\phi(y)$. Since $\phi$ is injective, $x=y$.

CoROllary. A manual $A$ is isomorphic to a submanual of a Hilbertian manual if and only if $A$ admits a unital, strongly separating set of amplitude states.

COROLlARY. A manual $A$ has an outcome preserving faithful interpretation in a Hilbertian manual if and only if $A$ admits a unital, strongly $\perp$-determining set of amplitude states.

COROLlARY. If a manual $A$ admits a unital set of amplitude states $\Delta$, then $A$ has an outcome preserving interpretation in a Hilbert space manual.

Corresponding results hold in the last corollary if $\Delta$ is separating or strongly $\perp$-determining.

\section{REFERENCES}

1. D. J. Foulis and C. H. Randall, Operational statistics. I. Basic concepts, J. Math. Phys. 13 (1972), 1667-1675.

2. - Empirical logic and quantum mechanics, Synthese 29 (1974), 81-111.

3. - The empirical logic approach to the physical sciences, in Foundations of Quantum Mechanics and Ordered Linear Spaces (A. Hartkämper and H. Neumann, Eds.), Springer-Verlag, New York, 1974.

4. - Manuals, morphisms, and quantum mechanics, in Mathematical Foundations of Quantum Theory (A. R. Marlow, Ed.), Academic Press, New York, 1978.

5. S. Gudder, Stochastic methods in quantum mechanics, North-Holland, New York, 1979.

6. J. M. Jauch, Foundations of quantum mechanics, Addison-Wesley, Reading, Mass., 1968.

7. G. Mackey, The mathematical foundations of quantum mechanics, Benjamin, New York, 1963.

8. C. H. Randall and D. J. Foulis, Operational statistics. II. Manuals of operations and their logics, J. Math. Phys. 14 (1973), 1472-1480.

9. - A mathematical setting for inductive reasoning, in Foundations of Probability Theory, Statistical Inference and Statistical Theories of Science (C. A. Hooker, Ed.), Reidel, Dordrecht, 1976.

10. - The operational approach to quantum mechanics, in The Logico-Algebraic Approach to Quantum Mechanics (C. A. Hooker, Ed.), Reidel, Dordrecht, 1977.

11. V. Varadarajan, Geometry of quantum theory, Vol. I, Van Nostrand-Reinhold, Princeton, N.J., 1968.

12. R. Wright, Spin manuals, empirical logic talks quantum mechanics, in Mathematical Foundations of Quantum Theory (A. R. Marlow, Ed.), Academic Press, New York, 1978.

DEPARTMENT OF MATHEMATICS AND COMPUTER SCIENCE, UNIVERSITY OF Denver, Denver, Colorado 80208 